\title{
Prolonged Stupor in Perampanel Overdose and Pharmacokinetic Considerations
}

Case Report

Joumal of Epilepsy Research pISSN 2233-6249 / eISSN 2233-6257

Received May 17, 2018

Revised September 18, 2018

Accepted October 1, 2018

Corresponding author:

Dong Wook Kim, MD, PhD

Department of Neurology, Konkuk

University School of Medicine, 120-1

Neungdong-ro, Gwangjin-gu, Seoul 05030,

Korea

Tel. $+82-2-2030-7460$

Fax. +82-2-2020-7460

E-mail; drdongwkim@kuh.ac.kr

\author{
Soojeong Kim, MD¹ , Tae-Eun Kim, MD, PhD² , Dayoung Kim, MD¹ , Dong Wook Kim, MD, PhD \\ Departments of ${ }^{1}$ Neurology, ${ }^{2}$ Clinical Pharmacology, Konkuk University School of Medicine, Seoul, Korea
}

\begin{abstract}
Perampanel is a new antiepileptic drug with a long half-life, but there are currently no data on the effects of acute perampanel overdose in epilepsy patients. We report a patient who had such an overdose. A 39-year-old woman was admitted for altered consciousness after taking 10 times the daily dose of perampanel. Her blood level of the drug was predicted, by pharmacokinetic calculation, to decrease to a steady-state level after 7 days, followed by a full recovery 8 days after the overdose. During her hospital stay, the patient experienced pulmonary embolism, which may be associated with the a-amino-3-hydroxy-5-methyl-4-isoxazolepropionic acid (AMPA) antagonistic mechanism of perampanel. The present findings show that perampanel overdose can produce prolonged stupor and that caution should be exercised during transient hypercoagulable states. (2018;8:87-89)
\end{abstract}

Key words: Perampanel, Overdose, Pharmacokinetics

\section{Introduction}

Perampanel is the first selective noncompetitive a-amino-3-hydroxy-5-methyl-4-isoxazolepropionic acid (AMPA) receptor antagonist for treating epilepsy. ${ }^{1}$ Its efficacy and tolerability as an adjuvant treatment in adults with drug-resistant focal seizure were demonstrated in three randomized, double-blind, placebo-controlled studies, and its efficacy as an adjuvant treatment for generalized seizure was also shown in a randomized study. ${ }^{2,3}$ The usual starting dose of perampanel for focal and generalized seizures in adult patients who are not concomitantly taking an enzyme-inducing antiepileptic drug (AED) is $2 \mathrm{mg} /$ day before sleep. Additionally, it is recommended that the dose be increased by $2 \mathrm{mg} /$ day biweekly to reach the maximum tolerable dose (usually 4-8 mg/day), which provides excellent seizure control. Among the currently available AEDs, perampanel has the longest metabolic half-life, up to 105 hours; thus, a theoretical steady-state can be reached in approximately 2-3 weeks after the drug is introduced or the dose adjusted. ${ }^{4}$

Perampanel treatment can be associated with various adverse effects. While the occurrence of psychiatric reactions is the most troublesome and often hinders the drug's widespread use in epilepsy patients, adverse dose-dependent central nervous system (CNS) effects such as dizziness, somnolence, and fatigue are the most frequently observed. Therefore, severe adverse CNS effects may occur in pa- tients with acute perampanel overdose and could be prolonged given the drug's long half-life. However, there is currently no report on the acute overdose of perampanel in epilepsy patients. We recently treated a patient who took 10 times the usual perampanel maintenance dose in an attempted suicide.

\section{Case}

A 39-year-old woman with an unremarkable medical history visited our neurology department complaining of recurrent seizures. Magnetic resonance imaging showed non-enhancing diffuse enlargement of the right amygdala, hippocampus, and parahippocampal gyrus, and electroencephalography showed occasional spikes in corresponding areas. The patient was initially prescribed $1,000 \mathrm{mg}$ of valproic acid once a day before sleep, but her seizures persisted. Two $\mathrm{mg} /$ day of perampanel before sleep was added as adjunctive therapy and up-titrated to $4 \mathrm{mg} /$ day after 2 weeks. Seizures were controlled with the combination therapy of $4 \mathrm{mg} /$ day of perampanel and 1,000 $\mathrm{mg} /$ day of valproic acid. After 11 months without seizures, the patient was admitted to the intensive care unit for altered consciousness. Her records documented that she had recently episode had a depression because of job loss and that she had taken 10 times the daily dosage of the prescribed AEDs ( $40 \mathrm{mg}$ of perampanel and 10,000 $\mathrm{mg}$ of valproic acid) in a suicide attempt. On neurological examination, her men- 
tal status was stuporous, but her reflexes were normal and there were no focal neurological signs or symptoms. Radiological evaluations, including brain computed tomography and diffusion-weighted magnetic resonance imaging, showed no abnormality, and continuous electroencephalography monitoring showed continuous low-amplitude slow activity without epileptiform discharges. Her blood valproic acid level on hospital day 2 exceeded $150 \mu \mathrm{g} / \mathrm{mL}$ and decreased to 88.39 and $3.44 \mu \mathrm{g} / \mathrm{mL}$ on day 3 and 7 , respectively. The patient's blood ammonia level on hospital day 3 was $125.2 \mu \mathrm{g} / \mathrm{dL}$ and decreased to $53.7 \mu \mathrm{g} / \mathrm{dL}$, within the reference range. Because it was impossible to measure the blood level of perampanel, we attempted to predict the patient's recovery time based on the known pharmacokinetics of perampanel (clearance, $0.75 \mathrm{~L} / \mathrm{h}$; volume of distribution, $100 \mathrm{~L}$; absorption rate constant, $\left.0.005 \mathrm{~h}^{-1}\right)$. We assumed that perampanel was regularly administered at a dosage of $4 \mathrm{mg} /$ day for 28 days to reach the steady-state level and that the $40 \mathrm{mg}$ had been taken at the 696th hour (day 29). We calculated that the blood perampanel level would increase up to twice the usual steady-state level but would decrease to the steady-state level at day 36 (7 days after she took $40 \mathrm{mg}$ of the drug; Fig. 1).

During the patient's hospital stay, vital signs and other laboratory results remained stable during the first 2 days, and there was no evidence of perampanel-induced systemic adverse effects such as hepatic or renal toxicity, hypotension, or respiratory suppression. However, we noted sudden respiratory difficulty on hospital day 3,

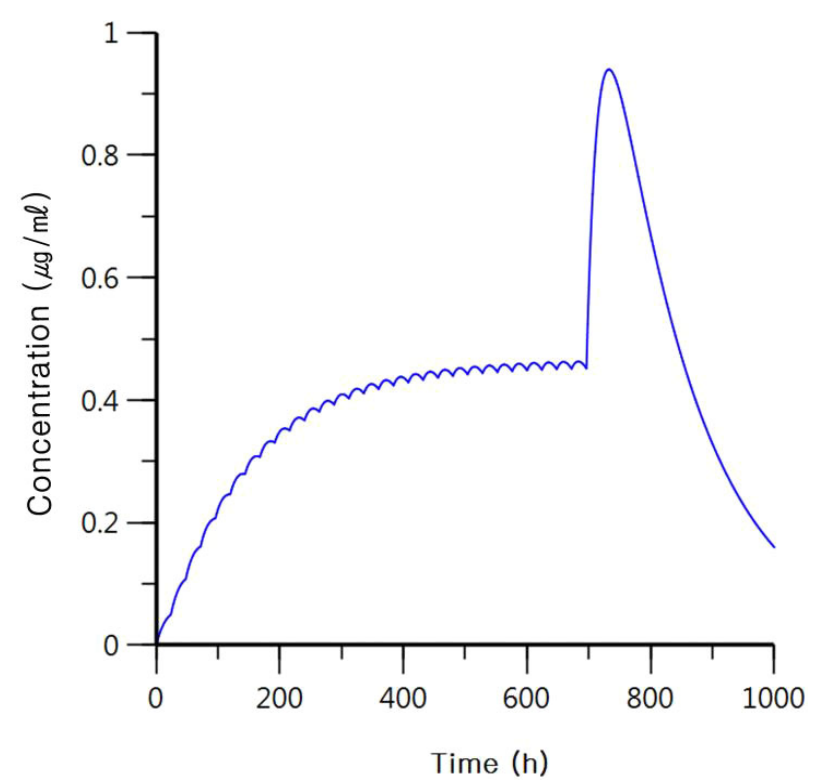

Figure 1. Calculated changes in blood perampanel levels after a patient took $40 \mathrm{mg}$ of the drug. and laboratory studies showed blood D-dimer levels exceeding $20 \mu \mathrm{g} / \mathrm{mL}$ and arterial hypoxia. Chest computed tomography also showed the development of a widespread pulmonary embolism. Under treatment with intravenous heparin anticoagulation and supportive mechanical ventilation, the patient began to regain consciousness on hospital day 6 , returning to her baseline mental status on day 8. Due to the possible adverse psychiatric effect of perampanel, which may have been associated with the suicide attempt, we recommended changing to another AED during the patient's hospital stay. However, the patient denied any causal relationship between the drug and the attempt and insisted that perampanel was effective for seizure control. At the 4-month follow-up at the outpatient clinic, she had remained seizure free with $4 \mathrm{mg} /$ day of perampanel and $100 \mathrm{mg} /$ day of valproic acid.

\section{Discussion}

We report here a patient who had a severe overdose of perampanel, 10 times the usual maintenance dose. Considering that the usual maintenance dose is 4-8 $\mathrm{mg} /$ day and the upper limit of the recommended daily dose is $12 \mathrm{mg} / \mathrm{day}{ }^{4}$ our patient had been administered a relatively low dose ( $4 \mathrm{mg} /$ day), and the dose she accidentally took (40 mg/day) was only 3.3 times higher than the upper limit of the recommended daily dose. However, it took approximately 1 week for her to recover consciousness, and this prolonged adverse CNS effect was likely associated with perampanel's long half-life. In previous reports of patients who had intoxication with other AEDs, systemic toxicity, including hyponatremia from oxcarbazepine, and cardiac arrhythmia from phenytoin, was occasionally observed, but patients recovered 2 or 3 days after admission., ${ }^{5,6}$ Our report shows that acute perampanel overdose may not produce serious adverse systemic effects, such as cardiac toxicity, respiratory depression, or other metabolic derangements, but that adverse CNS effects can be prolonged and that the recovery of consciousness may take longer in patients with high intoxication doses of the drug.

Psychiatric reactions are well-noted and troublesome adverse events of perampanel, and patients with perampanel treatment should be monitored for the occurrence of psychiatric reactions. Suicidal ideation and behavior have been reported in patients treated with AEDs on several occasions. A specific role of perampanel treatment in neuropsychiatric adverse effects is also suggested by observational studies. In a study of 47 individuals treated with a median perampanel dose of $8 \mathrm{mg} /$ day (range, 2-12 mg/day), behavioral alter- 
ations were the most frequent reason for discontinuation of treatment. In this cohort, three patients were reported to behave aggressively or experience suicidality. ${ }^{7}$

One remarkable finding was that our patient had pulmonary embolism during admission that necessitated anticoagulation therapy and mechanical ventilation. This was unexpected because AMPA receptors play an important role in modulating platelet activation and thrombosis, and AMPA receptor antagonists have been suggested for treating or preventing stroke, myocardial infarction, and other thrombotic diseases. ${ }^{8-11}$ The prolonged immobilization during hospital stay have been the main cause of pulmonary embolism in our patient, but it can also be speculated that the high blood level of perampanel during the acute overdose blocked the usual platelet activation; this might have induced a hypocoagulable state, and withdrawing the AMPA antagonist might have induced a transient hypercoagulable state that produced the embolism as a result of immobilization. In summary, these findings show that perampanel overdose can produce prolonged stupor due to its long half-life, and that caution should be exercised regarding the transient hypercoagulable state that could be associated with perampanel's effect on AMPA receptors.

\section{Conflicts of Interest}

No conflicts of interest have been declared.

\section{References}

1. Hanada T, Hashizume $\mathrm{Y}$, Tokuhara N, et al. Perampanel: a novel, orally active, noncompetitive AMPA-receptor antagonist that reduces seizure activity in rodent models of epilepsy. Epilepsia 2011;52:1331-40.

2. Rosenfeld W, Conry J, Lagae L, et al. Efficacy and safety of perampanel in adolescent patients with drug-resistant partial seizures in three double-blind, placebo-controlled, phase III randomized clinical studies and a combined extension study. Eur J Paediatr Neurol 2015;19:435-45.

3. French JA, Krauss GL, Wechsler RT, et al. Perampanel for tonic-clonic seizures in idiopathic generalized epilepsy a randomized trial. Neurology 2015;85:950-7.

4. Greenwood J, Valdes J. Perampanel (fycompa): a review of clinical efficacy and safety in epilepsy. P T 2016;41:683-98.

5. van Opstal JM, Janknegt $R$, Cilissen J, L'Ortije WH, Nel JE, De Heer F. Severe overdosage with the antiepileptic drug oxcarbazepine. $\mathrm{Br} J \mathrm{Clin}$ Pharmacol 2004;58:329-31.

6. Gaies E, Charfi R, Trabelsi S, Salouage I, Lakahl M, Klouz A. Acute phenytoin intoxication: two cases report and literature review. Therapie 2011; 66:461-3.

7. Coyle H, Clough P, Cooper R, Mohanraj R. Clinical experience with perampanel: focus on psychiatric adverse effects. Epilepsy Behav 2014;41: 193-6.

8. Weiser T. AMPA receptor antagonists for the treatment of stroke. Curr Drug Targets CNS Neurol Disord 2005;4:153-9.

9. Umemura K, Shimakura A, Nakashima M. Neuroprotective effect of a novel AMPA receptor antagonist, YM90K, in rat focal cerebral ischaemia. Brain Res 1997;773:61-5.

10. Catarzi D, Colotta V, Varano F. Competitive AMPA receptor antagonists. Med Res Rev 2007;27:239-78.

11. Morrell CN, Sun $H$, Ikeda $M$, et al. Glutamate mediates platelet activation through the AMPA receptor. J Exp Med 2008;205:575-84. 